\title{
Association of polymorphisms of the $D C N$ gene with growth traits in cattle (Brief Report)
}

\section{Assoziation des DCN-Gens mit Wachstumsmerkmalen beim Rind (Brief Report)}

\begin{abstract}
JIAJIE SUN ${ }^{1 *}$, CHUANWEN GU $^{1 *}$, CHUNLEI ZHANG ${ }^{1 *}$, CHUZHAO LEI $^{2}$, XINGTANG FANG ${ }^{1}$, QIJIANG JIN ${ }^{1}$, DANXIA CHEN ${ }^{1}$, XIUYING SHI ${ }^{1}$, YU DU ${ }^{1}$ and HONG CHEN ${ }^{1,2}$

'Institute of Cellular and Molecular Biology, Xuzhou Normal University, Xuzhou, People's Republic of China, ${ }^{2}$ College of Animal Science and Technology, Northwest A\&F University, Shaanxi Key Laboratory of Molecular Biology for Agriculture, Yangling, Shaanxi, People's Republic of China
\end{abstract}

\section{Background}

Mammalian decorin $(D C N)$ consists of a protein core and a single dermatan or chondroitin sulfate glycosaminoglycan chain (CHOPRA et al. 1985), contributing multifunctionally to processes like matrix assembly, modulation of the activity of growth factors and cell migration and proliferation. Recently, LINDA et al. (2008) have reported that eight SNPs were identified in human. However, the related information in bovine is scarce. Hence, in the present experiment the exons and intron/exon boundaries of DCN were scanned for SNPs in the predominant cattle breeds of China.

\section{Procedure}

Based on bovine DCN (GenBank acc. no. NC_007303), ten pairs of primers were designed to amplify the exons and intron/exon boundaries.
$D_{1}$ : F 5'-GGA GTA GAA GCA GGA GGT-3'
D2: F 5'-CAC ATA CAT TAG GCA AGG C-3'
R 5'-CCA AAT ACT TCG TTT CTG T-3'
R 5'-TCA CCC AGA TCA GAA CACT-3'
$D_{3}$ : F 5'-TTT AAT GAC TGC GTG TTG CT-3'
D4: F 5'-GAT GTT GCT TCT GTT CAC TA-3'
R 5'-GCTTTA CTC CAT CAC TCC CT-3'
R 5'-GAT TCA ATA CCC ATT TCT CC-3'
D5: F 5'-GCA GTT TCC TCA GGT TGT CC-3'
R 5'-ACC CGT GGC TGA TTC AAG TC-3'
D6: F 5'-ATG TAT TAT TGT AAA AGG GAT G-3'
R 5'-CAG CAG AAG TTT GTG GTT-3'
$D_{7}:$ F 5'-AAT CAC ATT AGG CAG AGG T-3'
D8: F 5'-GTT CAC CTG TAC GGT CTC C-3'
R 5'-CAC AGT AGG TAG TGG CTT T-3'
R 5'-AAC TGC ATA TTT GGC TTT A-3'
$D_{9}:$ F 5'-TTT CCC ACA TGA CTT ATT-3'
R 5'-TTA CAT AGC CTG GAT TGA-3'

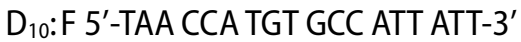
R 5'-ATA AGT CAT GTG GGA AAA-3'

A $15 \mu \mathrm{L}$ reaction mixture contained $50 \mathrm{ng}$ genomic DNA, $0.2 \mu \mathrm{M}$ of each primer, $1.5 \mathrm{mM}$ $\mathrm{MgCl}_{2}, 0.2 \mathrm{mM}$ dNTPs, and $0.60 \mathrm{U}$ Taq DNA polymerase (MBI). The cycling protocol was 5 min at $94^{\circ} \mathrm{C}, 32$ cycles of denaturing at $94^{\circ} \mathrm{C}$ for $45 \mathrm{~s}$, annealing at $X^{\circ} \mathrm{C}$ for 1 min, extension at $72^{\circ} \mathrm{C}$ for $45 \mathrm{~s}$, with a final extension at $72^{\circ} \mathrm{C}$ for $10 \mathrm{~min}$ ( $\mathrm{X}$ was $65,51,64,65,68,61,60,56$, 61 and 65 for $D_{1}, D_{2}, D_{3}, D_{4}, D_{5}, D_{6}, D_{7}, D_{8}, D_{9}$ and $D_{10}$ primers, respectively). The SSCP were analyzed according to previous description (PAN et al. 2007). The PCR products from individuals which represented different PCR-SSCP patterns were purified and sequenced. 


\section{Results}

SSCP polymorphisms were detected in $D_{2}, D_{3}, D_{5}$ and $D_{9}$ fragments of the bovine $D C N$ gene in 408 individuals (without genetic relationships) belonging to three Chinese genetic types: 68 Qinchuan cattle, 240 Nanyang cattle and 100 Jiaxian cattle. In the $D_{2}$ fragment, five SSCP genotypes were identified and designated as genotype $A_{2} A_{2}, A_{2} B_{2}, B_{2} B_{2}, A_{2} C_{2}$ and $B_{2} C_{2}$, respectively. The sequencing analysis of the five genotypes revealed two SNPs: g.[4204A $>G, 4264 A>G]$ in exon2 and they formed three consistent haplotypes: $A_{2}(A G)$, $B_{2}(G A)$ and $C_{2}(A A)$, respectively. Genotype $A_{2} A_{2}$ and haplotype $A_{2}$ were more prevalent and only Nangyang cattle significantly deviated from the Hardy-Weinberg equilibrium $(P<0.01)$. In the $D_{3}$ fragment, three SSCP genotypes were identified and named as genotype $A_{3} A_{3}, A_{3} B_{3}$ and $B_{3} B_{3}$, respectively. Only Qingchuan cattle were in HardyWeinberg equilibrium $(P>0.05)$. The sequence analysis revealed three SNPs g.[16476A>G, $16501 C>A, 16503 C>T$ ] . Through sequence comparison, haplotypes $A_{3}(G A T)$ and $B_{3}(A C C)$ were detected, respectively. In the $D_{5}$ locus, two unique SSCP banding patterns were observed and denominated as $A_{5} A_{5}$ and $A_{5} B_{5}$ genotypes. $A_{5}$ genotypes were predominant and only Nangyang cattle deviated from Hardy-Weinberg equilibrium $(P<0.01)$. The sequence analysis of the two genotypes revealed three SNPs g.[24200C >A, 24259T>G, $24285 G>A$ ]. They formed two consistent haplotypes $A_{5}(C T G)$ and $B_{5}(A G A)$. Three SSCP genotypes were identified in the $D_{9}$ fragment, which were denoted as genotype $A_{9} A_{9}, A_{9} B_{9}$ and $B_{9} B_{9}$, respectively. $A_{9}$ genotypes were more prevalent and genotype $C_{9}$ was only detected in the Jiaxian cattle. Consistent with the $D_{2}$ and $D_{5}$ fragments, only Nangyang cattle deviated from Hardy-Weinberg equilibrium $(P<0.05)$. By sequence analysis of the three genotypes a SNP g.38655G $>A$ was identified (alleles $A_{9}$ and $B_{9}$, respectively). The discovered sequences of alleles were deposited in GenBank (acc no. GQ249670 GQ249678). The relationships between polymorphic loci and growth traits in Nanyang cattle at 12 months old were analyzed by ANOVA using the following model:

$$
Y_{i j k l}=\mu+\text { breed }_{i}+\text { age }_{j}+\text { marker }_{k}+e_{i j k}
$$

where $Y_{i j k}$ is the observation of the trait, $\mu$ is the least square mean, breed ${ }_{i}$ is the effect of breed, $a e_{j}$ is the effect of age, marker $_{k}$ is the effect of marker genotype and $e_{i j k}$ is the residual effect. Significant differences between polymorphic loci and birth weight were identified and genotype $A_{2}, A_{3}, A_{5}$ and $A_{9}$ were obviously associated with higher values than the others $(P<0.01)$ (Table 1$)$. Hence, we firstly suggested that $D C N$ gene could be regarded as molecular marker for superior birth weight.

\section{Acknowledgements}

This study was supported by the National 863 Program of China (No. 2006AA10Z197, 2008AA101010), National Natural Science Foundation of China (No.30771544, 30972080), National Key Technology R\&D Program (No. 2006BAD01A10-5), Keystone Project of transfergene in China (2009ZX08009-157B, 2008ZX08007-002), »13115 " Sci-Tech Innovation Program of Shaanxi Province (2008ZDKG-11), MaTS-Beef Cattle System, Basic and Foreland Technology Study Program of Henan Province (No.072300430160), Natural science fund for colleges and universities in Jiangsu Province (09KJD180002). 
Table 1

Least square mean (means \pm standard error of means) of growth traits for the genotypes of polymorphic loci LSM (Mittel \pm Standardfehler) der Wachstumsmerkmale in Abhängigkeit von DCN

\begin{tabular}{llccccccc}
\hline \multicolumn{1}{c}{$\mathrm{G}(\mathrm{N})$} & $\mathrm{BiW}, \mathrm{kg}$ & $\mathrm{BoW}, \mathrm{kg}$ & $\mathrm{BH}, \mathrm{cm}$ & $\mathrm{BL}, \mathrm{cm}$ & $\mathrm{ChC}, \mathrm{cm}$ & $\mathrm{HW}, \mathrm{cm}$ & $\mathrm{ADG}, \mathrm{kg}$ \\
\hline & $\mathrm{A}_{2} \mathrm{~A}_{2}(156)$ & $29.2 \pm 0.2^{\mathrm{A}}$ & $222.6 \pm 1.8$ & $114.1 \pm 0.3$ & $116.8 \pm 0.6$ & $140.8 \pm 0.6$ & $20.6 \pm 0.1$ & $0.54 \pm 4.8$ \\
& $\mathrm{~A}_{2} \mathrm{~B}_{2}(72)$ & $29.1 \pm 0.4^{\mathrm{A}}$ & $226.4 \pm 3.0$ & $114.3 \pm 0.5$ & $118.2 \pm 1.0$ & $143.0 \pm 1.0$ & $20.9 \pm 0.2$ & $0.55 \pm 8.0$ \\
& $\mathrm{~B}_{2} \mathrm{~B}_{2}(5)$ & $25.6 \pm 0.7^{\mathrm{B}}$ & $228.0 \pm 7.1$ & $115.4 \pm 0.7$ & $120.6 \pm 3.0$ & $142.0 \pm 3.1$ & $20.3 \pm 1.2$ & $0.56 \pm 21.2$ \\
$\mathrm{D}_{2}$ & $\mathrm{~A}_{2} \mathrm{C}_{2}(2)$ & $24.8 \pm 1.3^{\mathrm{B}}$ & $242.0 \pm 18$ & $115.0 \pm 1.0$ & $126.0 \pm 8.5$ & $148.0 \pm 2.0$ & $21.0 \pm 1.0$ & $0.60 \pm 53.5$ \\
& $\mathrm{~B}_{2} \mathrm{C}_{2}(5)$ & $25.2 \pm 0.6^{\mathrm{B}}$ & $223.4 \pm 7.4$ & $111.6 \pm 1.6$ & $118.6 \pm 2.2$ & $146.4 \pm 4.9$ & $20.8 \pm 0.6$ & $0.56 \pm 20.2$ \\
& $P$ & 0.001 & 0.603 & 0.567 & 0.230 & 0.123 & 0.712 & 0.386 \\
& $\mathrm{~A}_{3} \mathrm{~A}_{3}(118)$ & $29.1 \pm 0.3^{\mathrm{A}}$ & $222.8 \pm 2.1$ & $113.9 \pm 0.4$ & $116.6 \pm 0.7$ & $140.7 \pm 0.7$ & $20.6 \pm 0.1$ & $0.54 \pm 5.6$ \\
$\mathrm{D}_{3}$ & $\mathrm{~A}_{3} \mathrm{~B}_{3}(102)$ & $29.5 \pm 0.3^{\mathrm{A}}$ & $224.1 \pm 2.3$ & $114.4 \pm 0.4$ & $117.8 \pm 0.7$ & $142.5 \pm 0.7$ & $20.8 \pm 0.2$ & $0.54 \pm 6.2$ \\
& $\mathrm{~B}_{3} \mathrm{~B}_{3}(20)$ & $25.6 \pm 0.4^{\mathrm{B}}$ & $230.8 \pm 5.1$ & $114.2 \pm 0.7$ & $120.5 \pm 1.8$ & $143.2 \pm 2.0$ & $20.7 \pm 0.4$ & $0.57 \pm 14.5$ \\
& $P$ & $<0.001$ & 0.352 & 0.526 & 0.075 & 0.150 & 0.706 & 0.098 \\
& $\mathrm{~A}_{5} \mathrm{~A}_{5}(170)$ & $29.2 \pm 0.2^{\mathrm{A}}$ & $222.3 \pm 1.7$ & $113.9 \pm 0.3$ & $116.5 \pm 0.5$ & $140.6 \pm 0.6$ & $20.6 \pm 0.1$ & $0.54 \pm 4.5$ \\
$\mathrm{D}_{5}$ & $\mathrm{~A}_{5} \mathrm{~B}_{5}(70)$ & $28.4 \pm 0.4^{\mathrm{B}}$ & $228.3 \pm 3.0$ & $114.6 \pm 0.5$ & $119.7 \pm 1.0$ & $144.3 \pm 1.0$ & $21.0 \pm 0.2$ & $0.56 \pm 8.1$ \\
& $P$ & 0.007 & 0.158 & 0.790 & 0.203 & 0.412 & 0.104 & 0.237 \\
& $\mathrm{~A}_{9} \mathrm{~A}_{9}(185)$ & $29.4 \pm 0.2^{\mathrm{A}} 222.7 \pm 1.6$ & $114.0 \pm 0.3$ & $116.6 \pm 0.5$ & $140.8 \pm 0.6$ & $20.7 \pm 0.1$ & $0.54 \pm 4.4$ \\
$\mathrm{D}_{9}$ & $\mathrm{~A}_{9} \mathrm{~B}_{9}(55)$ & $27.7 \pm 0.4^{\mathrm{B}}$ & $228.6 \pm 3.3$ & $114.7 \pm 0.5$ & $120.1 \pm 1.1$ & $144.4 \pm 1.0$ & $20.9 \pm 0.2$ & $0.56 \pm 8.9$ \\
& $P$ & $<0.001$ & 0.557 & 0.899 & 0.372 & 0.732 & 0.268 & 0.648 \\
\hline
\end{tabular}

G (N) genotype (observed number), BiW birth weight, BoW body weight, BH body height, BL body length, ChC chest circumference, HW hucklebone width, ADG average day gain. Treatments with different capital letters are significantly different at $P<0.01$.

\section{References}

Chopra RK, Pearson CH, Pringle GA, Fackre DS, Scott PG (1985) Dermatan sulphate is located on serine-4 of bovine skin proteodermatan sulphate. Biochem J 232, 277-9

Linda EK, Fergus JC, Shahana A, Alison MD (2008) Genetic variation in stromal proteins decorin and lumican with breast cancer: investigations in two case-control studies. Breast Cancer Res 10, R98

Pan CY, Lan XY, Chen H, Hua LS, Guo YK, Zhang B, Lei CZ (2007) Five novel single nucleotide polymorphisms (SNPs) of the prophet of PIT1 (PROP1) gene in bovine. Arch Tierz 50, 421-3

Received 30 November 2009, accepted 5 January 2010.

Corresponding author:

HONG CHEN

email: chenhong1212@263.net

Institute of Cellular and Molecular Biology, Xuzhou Normal University, Shanghai Road No. 101, Xuzhou, People's Republic of China 